\title{
STILI ATTRIBUTIVI MOTIVAZIONALI NELL'APPRENDIMENTO DELL'ITALIANO L1 - L2
}

\author{
Neala Ambrosi-Randić \\ Università Juraj Dobrila di Pola \\ Dipartimento di studi in lingua italiana
}

\section{Riassunto}

Le attribuzioni sono dei modi che le persone abitualmente usano per analizzare i propri successi o insuccessi. Il saggio presenta una ricerca sugli stili attributivi motivazionali nell'apprendimento dell'italiano come L1 e L2. Hanno preso parte alla ricerca 694 alunni dalla quinta all'ottava classe delle scuole elementari con insegnamento in lingua croata ( $\mathrm{n}$ $=453)$ e insegnamento in lingua italiana $(n=241)$. Mediante quattro domande scelte da un questionario sugli stili attributivi, gli alunni erano posti in situazioni virtuali di successo e insuccesso nei compiti di italiano fatti a casa e in classe. Dalle analisi risulta che la causa principale del successo è l'impegno, mentre l'insuccesso si attribuisce in primo luogo al disimpegno. I risultati ottenuti sono di buon auspicio, poiché lo stile attributivo più funzionale per la riuscita scolastica è appunto quello che riconosce nell'impegno e nel disimpegno le cause dei propri successi o insuccessi.

Parole chiave: motivazione, attribuzione, alunni, lingua italiana L1-L2

\section{Introduzione}

Nell'ambito della psicologia, la motivazione rappresenta un concetto ampio e complesso, definito, studiato e spiegato da teorie diverse. Le interpretazioni teoriche della motivazione ad apprendere sviluppate negli ultimi decenni offrono un panorama veramente vasto. Cercando di raggruppare i vari modelli, è possibile distinguere le teorie comportamentali che spiegano la motivazione usando i concetti di stimolo e risposta; quelle umaniste incentrate sui bisogni della persona; e le teorie cognitive che spostano l'attenzione sul ruolo dei processi cognitivi. In effetti, le teorie cognitive affermano che a muovere il comportamento umano è il significato che il soggetto attribuisce a un bisogno o a una situazione. In seguito ai vari eventi della vita, ogni individuo cerca di dare o trovare delle spiegazioni. Di fronte ad un successo o insuccesso si chiede che cosa può aver causato quel risultato. Le attribuzioni sono dei processi attraverso i quali ogni persona analizza i propri successi o insuccessi per capire chi o che cosa è responsabile della riuscita o del fallimento. Uno dei primi a proporre il concetto del locus di controllo fu Heider (1958), distinguendo gli eventi attribuiti a sé (impegno e abilità) da quelli esterni (difficoltà/facilità del compito, fortuna). In seguito, altri autori 
(Weiner, Frieze, Kukla, Reed, Rest, Rosenbaum, 1971) introdussero la dimensione della stabilità, importante poiché influenza i cambiamenti nelle aspettative della persona dopo un successo o un insuccesso. Weiner (1985) introdusse in questa classificazione la dimensione della controllabilità spiegando che è controllabile lo sforzo, ma non la fortuna o le abilità. Nella Tabella 1 presentiamo lo schema esaustivo delle tipologie di attribuzioni formulato da Weiner (1985): i vari successi o insuccessi sono principalmente attribuiti alle cause seguenti: la presenza o la mancanza di impegno, le proprie abilità, la facilità del compito, l'aiuto esterno e la fortuna. Le cause elencate fanno riferimento a tre dimensioni psicologiche diverse: il locus di causalità, la controllabilità e la stabilità. Il locus o luogo di causalità può essere esterno o interno, la controllabilità può essere presente (evento controllabile) o assente (evento incontrollabile), mentre per quanto riguarda la dimensione della stabilità distinguiamo tra cause stabili e instabili.

Tabella 1 - Le attribuzioni secondo Weiner

\begin{tabular}{ccc}
\hline Locus interno & Instabile controllabile & impegno / disimpegno \\
& Stabile incontrollabile & abilità / incapacità \\
\hline Locus esterno & Instabile controllabile & aiuto / non aiuto \\
& Stabile incontrollabile & facilità / difficoltà \\
& Instabile incontrollabile & fortuna / sfortuna \\
\hline
\end{tabular}

Le attribuzioni sono importanti, perché influenzano le prestazioni cognitive e l'apprendimento a scuola, la scelta del compito, la persistenza nel lavoro come pure le aspettative che si creano in merito all'esito del compito ed inoltre le emozioni. In seguito ad un successo nelle attività o compiti scolastici gli alunni possono presentare spiegazioni diverse di quanto accaduto. Alcuni attribuiscono il proprio successo all'impegno usato nella realizzazione del compito. L'impegno è considerato una causa interna perché dipende dal soggetto; instabile poiché può esserci o non esserci (in alcune situazioni si decide di impegnarsi poco, mentre in altre abbastanza o molto); controllabile giacché è l'individuo il principale responsabile del proprio impegno e può decidere se, quanto $\mathrm{e}$ come impegnarsi. Altri invece, di fronte alla stessa situazione, attribuiscono il proprio successo a un'altra causa. La fortuna è considerata una causa esterna al soggetto, che non dipende da lui, instabile e incontrollabile perché può esserci o non esserci, ma non dipende dal soggetto (Ravazzolo, De Beni, Moè, 2005).

Le spiegazioni che l'individuo usa di fronte ai propri successi o insuccessi sono tendenzialmente stabili e derivano da uno schema personale che si mantiene nel corso del tempo. La scelta della causa per spiegare i propri successi o insuccessi influenza l'apprendimento scolastico, poiché incide sulla probabilità di usare strategie adatte per risolvere un compito o problema. Weiner (1972) e i suoi collaboratori sono riusciti a dimostrare che le attribuzioni, definite come reazioni individuali al successo e al fallimento, possano permettere di predire il comportamento orientato al successo.

Lo stile attributivo più funzionale per la riuscita nella vita e nell'ambito scolastico è quello che riconosce nell'impegno o nella mancanza di impegno la causa principale dei propri successi o insuccessi. Numerose sono le ricerche che hanno dimostrato il 
collegamento di questo stile con altre variabili: una maggior tendenza e perseguire obiettivi di padronanza piuttosto che di prestazione (Ames, 1992); superiori livelli di persistenza nella soluzione di compiti impegnativi (Dweck, 2000); aumento e mantenimento della motivazione (Moè, De Cal, De Beni, 2002); un maggiore senso di autoefficacia (Bandura, 2000); una migliore riuscita scolastica (Henry, Martinko, Pierce, 1993).

La presente ricerca si colloca all'interno delle teorie cognitive della motivazione, e in modo specifico parte dalla teoria delle attribuzioni causali. Per esplorare lo stile attributivo nel nostro campione di ricerca abbiamo usato delle domande che ci hanno premesso di analizzare il modo in cui gli alunni compiono le attribuzioni del proprio successo o insuccesso nell'apprendimento dell'italiano come lingua dell'ambiente (L1) o lingua seconda (L2).

\section{Metodologia della ricerca}

\subsection{Procedimento}

La ricerca fa parte di un progetto più ampio (La motivazione nell'apprendimento/ insegnamento dell'italiano nell'Istroquarnerino), finanziato con i mezzi del Ministero degli affari esteri della Repubblica Italiana e condotto nel periodo dal 2008 al 2010. Una considerevole parte dei dati è stata pubblicata nel volume L'italiano L2: indagini sulla motivazione (Scotti Jurić, Ambrosi-Randić, 2010), mentre quelli presenti in questo saggio sono di prima pubblicazione.

\subsection{Strumenti}

Per misurare lo stile attributivo degli alunni abbiamo scelto quattro domande tratte dalla versione semplificata del Questionario per studenti dagli 11 ai 20 anni di De Beni e Moè (1995). Il questionario è originariamente composto da 24 item che presentano delle situazioni ipotetiche in cui l'alunno potrebbe essersi trovato, seguito dalle cinque possibili cause attribuibili a quell'evento. Dodici item riguardano situazioni di successo e dodici di insuccesso. Nella nostra ricerca ci siamo limitati a due situazioni di successo e due di insuccesso. Per ogni serie, nella versione originale del questionario, troviamo quattro che riguardano situazioni di apprendimento, quattro di memoria e quattro di vita quotidiana. In sintonia con i nostri obiettivi e finalità della ricerca ci siamo valsi soltanto degli item che comprendevano le situazioni di apprendimento compiendo una lieve modifica e cioè facendo riferimento non all'apprendimento scolastico in generale, ma in modo specifico all'apprendimento dell'italiano. La domanda tipica è: In un compito di italiano hai eseguito tutti gli esercizi correttamente. Perché è successo questo? Come modalità di risposta a questa domanda, gli alunni potevano scegliere una delle cinque spiegazioni elencate qui di seguito: sono stato fortunato/ta; ce l'ho messa tutta; sono stato bravo/a; era facile; e sono stato aiutato/a. Per gli alunni delle scuole elementari con insegnamento in lingua italiana è stato usato il questionario in italiano, mentre per quelli delle scuole elementari croate lo stesso questionario in lingua croata. 


\subsection{Partecipanti}

Il campione preso in esame per questa ricerca è composto da 453 alunni delle scuole elementari con insegnamento in lingua croata e 241 alunni delle scuole elementari con insegnamento in lingua italiana, per un totale di 694 alunni. Si tratta di 311 ragazzi $(44,8 \%)$ e 383 ragazze $(55,2 \%)$ che studiano l'italiano come seconda lingua (L2) nel caso delle scuole con insegnamento in lingua croata e lingua dell'ambiente sociale (L1) per gli alunni che frequentano le scuole con insegnamento in lingua italiana. L'età degli alunni era compresa tra gli undici e quindici anni con una media di 12.26 (DS = 2.19). Sono stati indagati gli alunni dalla quinta all'ottava classe delle scuole di: Albona, Buie, Fiume, Parenzo, Pisino, Pola, Rovigno e Umago.

\section{Risultati e discussione}

I dati ottenuti sulle attribuzioni degli alunni che apprendevano l'italiano quale L1 o L2 figurano nei grafici successivi. Le risposte degli alunni sotto forma di percentuali figurano nei grafici 1-4, mentre le frequenze delle rispettive risposte sono state usate per calcolare il chi-quadrato riportato nelle note. Nel Grafico 1 troviamo i risultati sull'attribuzione del proprio successo in una situazione scolastica. La maggior parte degli alunni delle elementari (45\% e 46\%) attribuisce il proprio successo nei compiti di italiano all'impegno. Indipendentemente dal fatto se si tratta dell'italiano come L1 o L2 le attribuzioni del successo sono uguali. ${ }^{1}$ Mentre la maggioranza attribuisce il successo al proprio impegno, al secondo posto figura la facilità del compito. Le due cause principali scelte per spiegare il proprio successo (impegno e facilità) sono fondamentalmente diverse poiché la prima causa è instabile e controllabile, mentre la seconda è stabile e incontrollabile. Da queste attribuzioni di conseguenza derivano comportamenti diversi.

Grafico 1 - Attribuzione del successo nei compiti di italiano

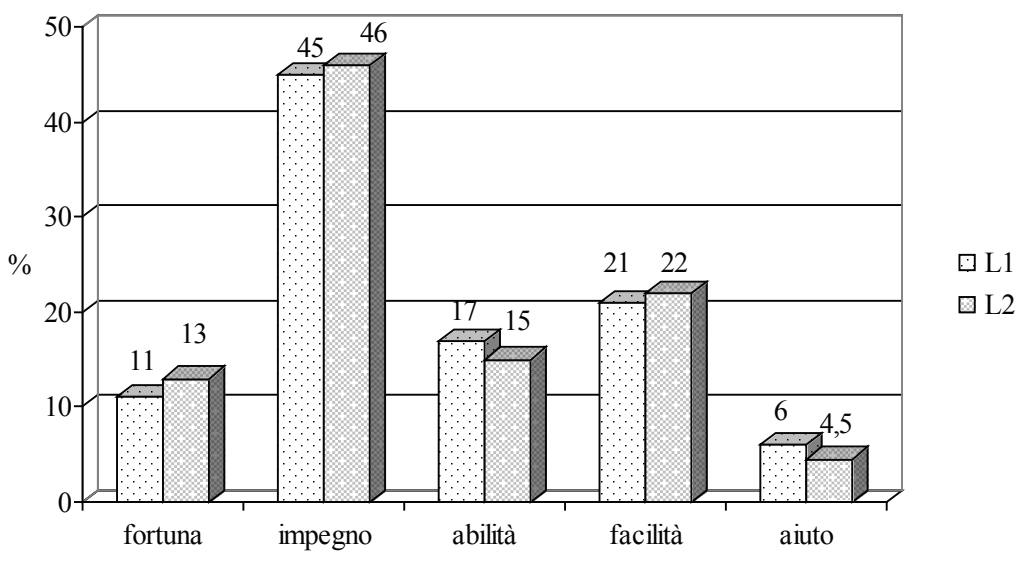

$1 \quad \chi^{2}=2,2 ; \mathrm{gl}=4, \mathrm{p}=\mathrm{n} . \mathrm{s}$. 
Per quanto riguarda l'attribuzione dell'insuccesso nei compiti di italiano svolti a casa (Grafico 2), la metà degli alunni, sia delle elementari croate sia di quelle italiane, nomina il disimpegno, seguito poi dalla difficoltà del compito e dalla mancanza di abilità. Le attribuzioni dell'insuccesso nei due gruppi sono diverse ${ }^{2}$, poiché nell'apprendimento della L2 un numero significativamente più alto di alunni attribuisce l'insuccesso alla difficoltà del compito (32\%) la quale viene nominata soltanto dal $18 \%$ degli alunni della L1; mentre vale l'opposto per la mancanza di abilità che viene più spesso nominata dagli alunni della L1 rispetto a quelli della L2. Inoltre, da rilevare pure le differenze che riguardano la sfortuna: nominata come causa dell'insuccesso più spesso nel caso della L1 (10\%) e quasi assente nell'apprendimento della L2.

Grafico 2 - Attribuzione dell'insuccesso nei compiti a casa

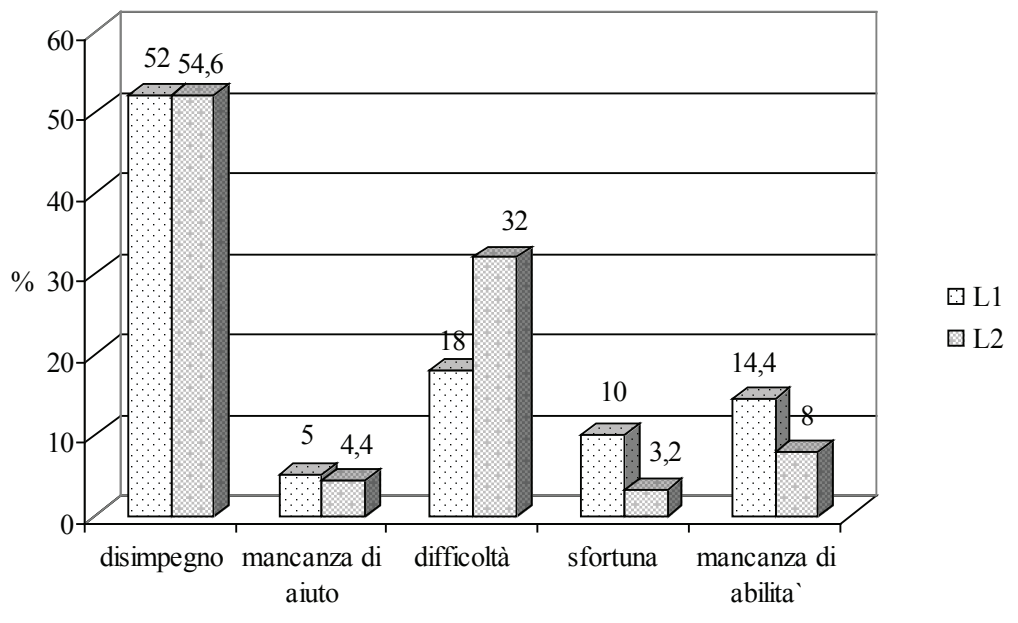

Nel Grafico 3 figurano le attribuzioni del successo nella presentazione verbale in classe. La prima causa nominata è uguale per tutti (l'impegno) ${ }^{3}$, ma va notata la considerevole differenza tra gli alunni della L1 e L2. L'impegno come causa del successo è nominato dal $63 \%$ degli alunni della L2 e da una percentuale considerevolmente più bassa (49\%) di alunni della L1. La seconda causa del successo per gli alunni della L1 si trova nell'abilità, mentre per gli alunni della L2 al secondo posto con percentuali pressoché uguali troviamo non una, ma tre spiegazioni diverse: facilità, abilità e fortuna.

Paragonando e analizzando i Grafici 1 e 3 che fanno riferimento alle situazioni di successo è interessante notare che con il cambiamento della tipologia del compito, non cambia l'attribuzione della prima causa del successo. Per gli alunni delle scuole elementari nell'apprendimento della L1 e L2 la causa principale del successo resta sempre uguale, l'impegno, anche quando cambia il compito.

$2 \quad \chi^{2}=30,11 ; \mathrm{gl}=4, \mathrm{p}<.001$

$3 \quad \chi^{2}=21,3 ; \mathrm{gl}=4, \mathrm{p}<.001$ 
Grafico 3 - Attribuzione del successo nella presentazione verbale

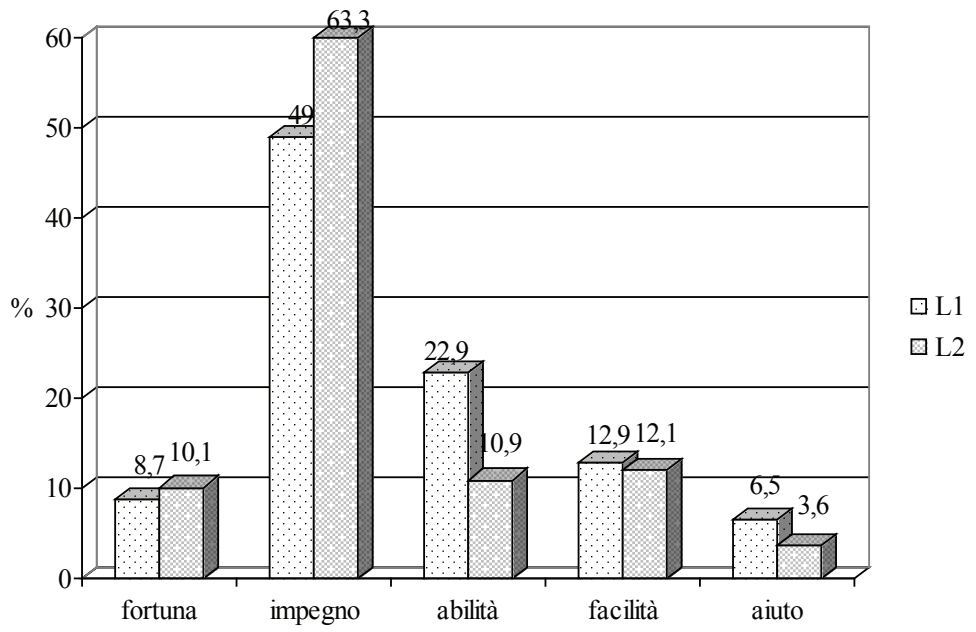

Nell'attribuzione dell'insuccesso negli esercizi di italiano (Grafico 4) non ci sono differenze significative tra gli alunni della L1 e quelli della L2. ${ }^{4}$ Quale causa principale dell'insuccesso figura il disimpegno, seguita dalla difficoltà del compito stesso.

Le attribuzioni dell'insuccesso (Grafico 2) nei compiti di italiano fatti a casa e successo nella presentazione verbale (Grafico 3) sono significativamente diverse tra gli alunni della L1 e L2, mentre non risultano diverse le attribuzioni del successo e insuccesso nell'esercizio di italiano (Grafici 1 e 4). Appare evidente come nel processo di attribuzione riguardante situazioni contraddistinte dal successo/insuccesso vi siano fattori ritenuti fuori dal nostro controllo (ad esempio la difficoltà del compito), e altri invece considerati come tendenzialmente costanti e controllabili.

Grafico 4 - Attribuzione dell'insuccesso nell'esercizio di italiano

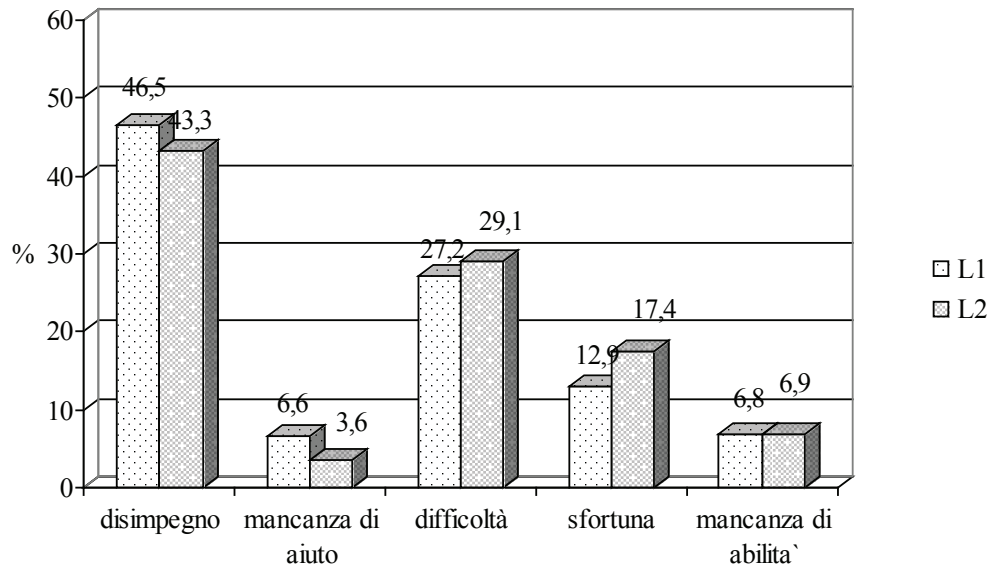

$4 \quad \chi^{2}=4,91 ; \mathrm{gl}=4, \mathrm{p}=$ n.s. 
Dalla presente ricerca emerge un quadro di attribuzioni causali molto interessante e di buon auspicio per la riuscita scolastica: come causa principale del successo/insuccesso gli alunni nominano l'impegno/disimpegno. È una causa instabile, ma controllabile, che indica l'esistenza di un locus di controllo interno. Stando alle varie ricerche nominate nella parte introduttiva di questo saggio, lo stile attributivo più funzionale per la riuscita nella vita e nell'ambito scolastico è appunto quello che riconosce nell'impegno o nella sua mancanza la causa principale dei propri successi o insuccessi. Le attribuzioni espresse rappresentano interpretazioni importanti, che permettono di capire il comportamento in futuro: se un individuo riporta un insuccesso e lo attribuisce alla propria scarsa abilità (causa stabile), la sua aspettativa di successo futuro diminuisce rispetto alla situazione in cui attribuisce il risultato a cause instabili, come la fortuna e l'umore del momento. Analogamente, il successo attribuito alla fortuna (causa instabile) fa aumentare meno l'aspettativa di successo futuro rispetto alla situazione in cui questo viene attribuito a cause stabili come l'abilità o la facilità del compito. I cambiamenti nelle aspettative, per l'esito futuro in un compito, dipendono dunque dalla stabilità delle cause cui il risultato ottenuto viene attribuito; in altre parole, se le cause del risultato vengono percepite come stabili, allora il successo (o il fallimento) futuro sarà anticipato dall'individuo con un maggior grado di certezza.

Considerata l'importanza del corretto riconoscimento dell'impegno come causa principale dei propri successi o insuccessi, nei libri di testo e varie altre fonti è possibile trovare sempre più spesso il consiglio volto ai genitori e insegnanti, di promuovere $\mathrm{e}$ sostenere un adeguato sviluppo dello stile attributivo. Questo intervento va compiuto quotidianamente, dopo ogni compito o attività, invitando l'alunno a riflettere su quanto accaduto e a riconoscere principalmente nel proprio impegno la causa dei successi o insuccessi. Molto utili a questo proposito sono i vari programmi sotto forma di interventi strutturati come quelli proposti da Ravazzolo e coll. (2005), Perticone (2004) e altri.

\section{Conclusione}

Per potenziare la motivazione nell'apprendimento, è compito dell'insegnante ampliare le esperienze dell'alunno, applicare strategie in situazioni in cui può essere utile ed esprimere sistematicamente un feedback di incoraggiamento. L'obiettivo principale dell'intervento è creare nell'alunno l'idea della relazione che esiste fra il suo impegno e i risultati ottenuti nei compiti assegnati. 


\section{BIBLIOGRAFIA}

AMES, C., 1992, Classroom: goals, structures and student motivation, in "Journal of Educational Psychology", 87, pp. 49-74.

BANDURA, A., 1986, Social foundations of thought and action; a social cognition theory, Englewood Cliff, Prentice-hall, New York.

BANDURA, A., 2000, Autoefficacia. Teoria e implicazioni, Erickson, Trento.

DE BENI, R., MOĖ, A., 1995, Questionario di attribuzione. Attribuzione delle cause di successo/fallimento in compiti cognitivi, Organizzazioni Speciali, Firenze.

DE BENI, R., MOĖ, A., 2000, Motivazione e apprendimento, Il Mulino, Bologna.

DWECK, C. S., 1986, Motivational processes affecting learning, in "American Psychologist", 41, pp. 1040-1048.

DWECK, C. S., 2000, Teorie del sé. Intelligenza, motivazione, personalità e sviluppo, Erickson, Trento Wiley.

HEIDER, F., 1958, The psychology of interpersonal relations, New York:

HENRY, J. W., MARTINKO, M. J., PIERCE, M. A., 1993, Attributional styles as a predictors of Success and Failure in a First Computer Course, in "Computers in Human Behavior", 9, pp. 341-352.

PERTICONE, G., 2004, La mia motivazione. Attività per sviluppare la voglia di apprendere nella scuola primaria, Erickson, Trento.

RAVAZZOLO, C., DE BENI, R., MOÈ, A., 2005, Stili attributivi motivazionali, Erickson, Trento.

SCOTTI JURIĆ, R., AMBROSI-RANDIĆ, N., 2010, L'italiano L2: indagini sulla motivazione. Pietas Iulia, Edit, Pola-Fiume.

WEINER, B., FRIEZE, I., KUKLA, A., REED, L., REST, S., \& ROSENBAUM, R., 1971, Perceiving the cause of success and failure, New York: General Learning Press.

WEINER, B., 1972, Theories of motivation: From mechanism to cognition, Chicago: Markham.

WEINER, B., 1985, An attributional theory of achievement motivation and emotion, in "Psychological Review", 92, pp. 548-573.

WEINER, B., 2005, Motivation from an attribution perspective and the social psychology of perceived competence, in ELLIOT, A. J., DWECK, C. S., (a cura di), Handbook of competence and motivation, 73-84, London, Guliford, New York. 


\section{ATRIBUCIJSKI STILOVI U MOTIVACIJI ZA UČENJEM TALIJANSKOGA KAO JEZIKA SREDINE I KAO STRANOGA JEZIKA}

Atribucije predstavljaju objašnjenja kojima osobe pripisuju vlastite uspjehe i neuspjehe. Rad predstavlja istraživanje o atribucijskim stilovima učenika pri učenju talijanskoga jezika kao jezika sredine odnosno stranoga jezika. U istraživanju je sudjelovalo 694 učenika, polaznika od petoga do osmoga razreda osnovnih škola s nastavom na hrvatskom jeziku $(\mathrm{n}=453)$ i nastavom na talijanskom jeziku $(\mathrm{n}=$ 241). Učenici su stavljeni u situaciju doživljavanja uspjeha i neuspjeha pri rješavanju zadataka iz talijanskoga jezika u školi i kod kuće pitanjima koja su sastavni dio upitnika o atribucijskim stilovima. Rezultati pokazuju da su učenici kao glavni razlog uspjeha navodili trud, a neuspjeha izostanak truda. Dobiveni su rezultati ocijenjeni vrlo povoljnima za ishode učenja jer je upravo atribucijski stil koji se pokazao najefikasnijim za postizanje školskoga uspjeha onaj koji prepoznaje trud kao uzrok uspjeha, odnosno izostanak truda kao razlog neuspjeha. Takve su atribucije još poznate kao uspješne atribucije.

Ključne riječi: motivacija, atribucije, učenici, talijanski jezik L1-L2

\section{ATTRIBUTION STYLES IN MOTIVATION FOR LEARNING THE ITALIAN LANGUAGE AS L1 OR L2}

Attributions represent explanations that people use for their personal success and failure. The paper presents a research of students' attribution styles in learning Italian as a language of the environment and Italian as a second language. 694 pupils from fifth to eighth class of primary school took part in the research. 453 of them were from schools with lessons in Croatian and 241 of them were from schools with lessons in the Italian language. They were put in situations of success and failure in tasks in the Italian language, both at school and at home, by using some questions from the attribution style questionnaire. Analysis showed that students recognized their effort as the primary cause of personal success and lack of effort as the primary cause of failure. Obtained results are very favourable for learning outcomes because attributions, previously identified as the most useful for school achievement, recognize effort as the cause of success and the lack of effort as the cause of failure. Such attributions were known as successful attributions.

Key words: motivation, attributions, students, Italian language L1-L2 